\title{
Right Supramarginal Gyrus Is Crucial to Overcome Emotional Egocentricity Bias in Social Judgments
}

\author{
Giorgia Silani, ${ }^{1,3 \star}$ Claus Lamm, ${ }^{2,3 *}$ Christian C. Ruff, ${ }^{3}$ and Tania Singer ${ }^{3,4}$ \\ ${ }^{1}$ Cognitive Neuroscience Sector, International School for Advanced Studies, SISSA-ISAS, 34136, Trieste, Italy, ${ }^{2}$ Social, Cognitive and Affective Neuroscience \\ Unit, Department of Basic Psychological Research and Research Methods, Faculty of Psychology, University of Vienna, 1010 Vienna, Austria, ${ }^{3}$ Laboratory \\ for Social and Neural Systems Research (SNS-Lab), Department of Economics, University of Zurich, CH-8006 Zurich, Switzerland, and ${ }^{4}$ Max Planck \\ Institute for Human Cognitive and Brain Sciences, Department of Social Neuroscience, 04103 Leipzig, Germany
}

Humans tend to use the self as a reference point to perceive the world and gain information about other people's mental states. However, applying such a self-referential projection mechanism in situations where it is inappropriate can result in egocentrically biased judgments. To assess egocentricity bias in the emotional domain (EEB), we developed a novel visuo-tactile paradigm assessing the degree to which empathic judgments are biased by one's own emotions if they are incongruent to those of the person we empathize with. A first behavioral experiment confirmed the existence of such EEB, and two independent fMRI experiments revealed that overcoming biased empathic judgments is associated with increased activation in the right supramarginal gyrus (rSMG), in a location distinct from activations in right temporoparietal junction reported in previous social cognition studies. Using temporary disruption of rSMG with repetitive transcranial magnetic stimulation resulted in a substantial increase of EEB, and so did reducing visuo-tactile stimulation time as shown in an additional behavioral experiment. Our findings provide converging evidence from multiple methods and experiments that rSMG is crucial for overcoming emotional egocentricity. Effective connectivity analyses suggest that this may be achieved by early perceptual regulation processes disambiguating proprioceptive first-person information (touch) from exteroceptive third-person information (vision) during incongruency between self- and other-related affective states. Our study extends previous models of social cognition. It shows that although shared neural networks may underlie emotional understanding in some situations, an additional mechanism subserved by rSMG is needed to avoid biased social judgments in other situations.

\section{Introduction}

Self-projection and simulation are important mechanisms for interpersonal understanding, as suggested by findings that shared neural activations between self and other underlie our ability to represent the internal states of others (Bastiaansen et al., 2009; Mitchell, 2009; Singer and Lamm, 2009). However, interpersonal misunderstandings and conflict can arise when such a self-referential projection mechanism is applied in situations where it is inappropriate; for example, when falsely assuming that someone else is happy or sad, just because we are. The tendency to project one's own mental

\footnotetext{
Received April 8, 2013; revised Aug. 5, 2013; accepted Aug. 15, 2013.

Author contributions: G.S., C.L., and T.S. designed research; G.S., C.L., and C.C.R. performed research;G.S. and C.L. analyzed data; G.S., C.L., C.C.R., and T.S. wrote the paper.

This research was funded by the Swiss National Science Foundation ("Neuronal and developmental basis of empathy and emotion control: fMRI studies of adults and children aged 6-12 years" to T.S.), the European Community's Seventh Framework Program (FP7/2007-2013/ERC Grant agreement no. 205557 to T.S.), the University Research Priority Programs to the University of Zurich, and the NCCR Affective Sciences (to C.C.R.), and the Viennese Science and Technology Fund (WWTF, CS11-016 to C.L.). We thank Romana Snozzi for help in developing the paradigm, Marco Zanon for collecting part of the data, Nikolaus Steinbeis for useful comments on an earlier version of the paper, and Christoph Eisenegger for support with the TMS experiment.

The authors declare no competing financial interests.

${ }^{*}$ G.S. and C.L. contributed equally to this work.

This article is freely available online through the J Neurosci Author Open Choice option.

Correspondence should be addressed to Dr. Tania Singer, Max Planck Institute for Human Cognitive and Brain Sciences, Department of Social Neuroscience, Stephanstr 1A, 04103 Leipzig, Germany. E-mail: singer@cbs.mpg.de. DOI:10.1523/JNEUROSCI.1488-13.2013

Copyright $\odot 2013$ the authors $\quad 0270-6474 / 13 / 3315466-11 \$ 15.00 / 0$
}

states onto others has broadly been referred to as egocentricity bias. Although considerable behavioral research has been devoted to egocentricity in the cognitive domain (Royzman et al., 2003; Pronin, 2008), hardly anything is known about biased judgments in the affective domain. Initial insights have been provided by developmental research focusing on social attribution of desires (Repacholi and Gopnik, 1997), and by showing that people's visceral states affect how they judge those states in others (Van Boven and Loewenstein, 2003; O'Brien and Ellsworth, 2012). Experimental paradigms allowing the specific investigation of emotional egocentricity bias (EEB) are absent, and the neural mechanisms underlying this bias remain fully unexplored.

To quantify EEB and to investigate our ability to overcome it, we therefore developed a new experimental paradigm, and used it in a series of five interwoven behavioral, fMRI, and transcranial magnetic stimulation (TMS) experiments. Our paradigm evoked congruent or incongruent emotions between a participant and another person by means of pleasant or unpleasant visuo-tactile stimulation. In some trials, participants had to provide empathic judgments of the emotions felt by the other person. During incongruent trials, this made it necessary to disregard their own emotional state. If these trials showed a shift in the empathic judgment of the other person's emotion toward the participant's own emotion, we could infer the presence of EEB and quantify its degree.

An initial behavioral experiment using this approach could indeed establish a significant EEB in healthy young adults, but 
a

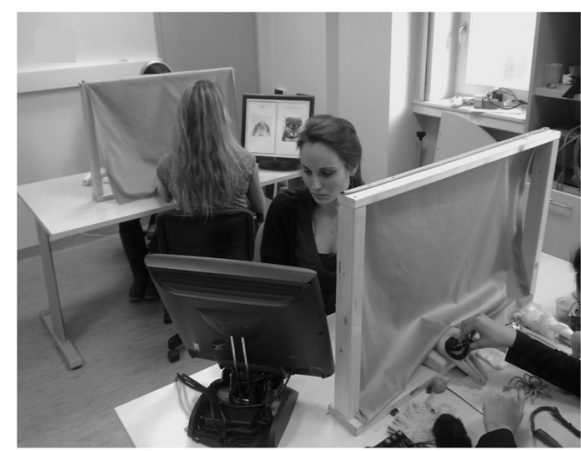

C

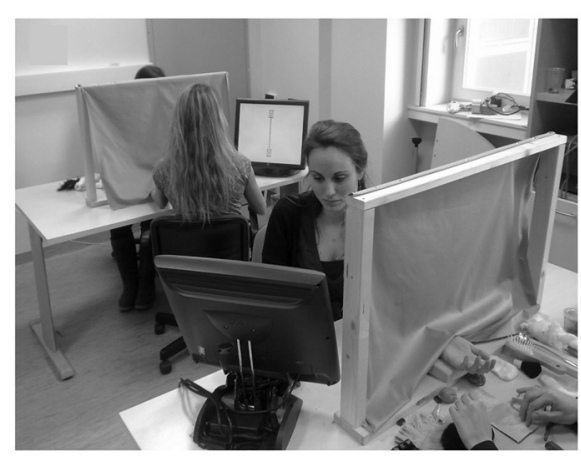

b

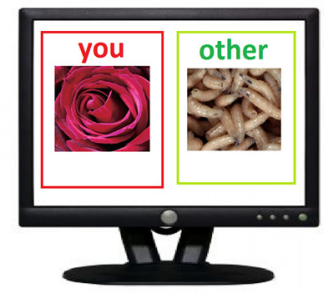

d

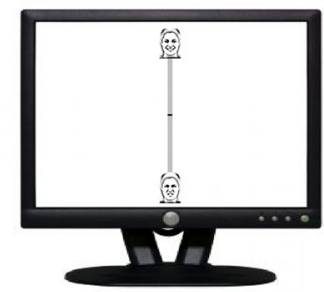

Figure 1. Setup for the behavioral experiment and examples of screen displays. $\boldsymbol{a}, \boldsymbol{c}$, Setup for the session, showing on the screen in $\boldsymbol{a}$ the simultaneous display of objects indicating self- and other-related stimulation, and in c the rating scale. $\boldsymbol{b}$, Screenshot of a trial entailing pleasant stimulation for the participant (rose), and unpleasant stimulation for the other person (maggots). $\boldsymbol{d}$, Screenshot of the visual analog scale used to deliver emotion judgments.

also revealed that the participants in our study seemed to be rather proficient in minimizing biased judgments. This led us to our second goal, the investigation of the neural mechanisms allowing humans to overcome such egocentric judgments. Some predictions concerning the neural structures related to this function could be derived from social cognitive neuroscience research focusing on self- other distinction in the cognitive and motor domain. This research converged on the importance of a region referred to as the right temporoparietal junction (rTPJ; Decety and Lamm, 2007; Van Overwalle, 2009, for meta-analyses). As rTPJ encompasses a fairly large area at the intersection of the posterior temporal and the inferior parietal cortex, we aimed to localize and characterize the specific roles of rTPJ and adjacent areas for EEB. This was achieved by two independent $\mathrm{fMRI}$ experiments, and by comparing their activations to meta-analyses of related functions requiring self-other distinction.

A final aim of our study was to investigate how overcoming EEB can be impaired. To this end, we used repetitive TMS (rTMS) to temporarily interfere with the normal functioning of the brain region identified by the fMRI experiments. The rTMS experiment was also crucial to inform us about the causal role of this area and whether its function was related to instantiating versus overcoming egocentric social judgments. Furthermore, an additional behavioral experiment confirmed the robustness of the EEB and tested whether our ability to overcome it also deteriorates when reducing the time available for its regulation.

\section{Materials and Methods}

We report the results of five related experiments with a total sample size of 191 healthy participants. As a first step, we performed a behavioral experiment $(N=72)$ to establish the experimental procedures to assess $\mathrm{EEB}$, and to estimate its size in healthy adults. Second, we performed an

exploratory "pilot" fMRI experiment $(N=$ 18), followed by a second fMRI experiment $(N=32)$. The results of the fMRI experiments were compared with activation related to attention reorienting, as well as quantitative meta-analyses of published findings related to theory of mind (Mar, 2011), agency (Sperduti et al., 2011), and imitation inhibition (Brass et al., 2009). Third, to investigate how EEB can be increased, a TMS experiment $(N=45)$ was used to determine the causal involvement of the brain area identified by the two fMRI experiments. Finally, we performed another behavioral replication experiment $(N=24)$ in which we explored whether the size of EEB can also be increased by a slight modification of the newly developed experimental paradigm. All experiments were performed with independently recruited samples of right-handed female undergraduate students (i.e., no participant had participated in any of the other experiments).

\section{Behavioral Experiment 1}

The aim of the initial behavioral experiment was to establish and validate the experimental procedures of our new paradigm, and to estimate the size of EEB. As in all experiments, participants were unknown to each other and assigned pairwise to an experimental session. They sat in front of a touch screen, placed their left hand under a curtain preventing them to observe their tactile stimulation, and were seated back to back to prevent that they directly observed each other's emotional responses or stimulation (Fig. 1). After familiarization with the stimuli and the rating procedure during a short practice trial, participants were presented with pictures of two objects (one for each participant) on the left and right side (position counterbalanced across participants) of a screen $(336 \times 336$ pixels at $800 \times 600$ pixels resolution, 15 inch screen, viewing distance $\sim 40 \mathrm{~cm}$ ). Visual presentation was accompanied in both participants by simultaneous stroking of the left hand at $1 \mathrm{~Hz}$ for $3 \mathrm{~s}$, with a material resembling the feel of the object depicted on the screen. The labels "You" and "Other" above the picture indicated the correspondence between the pictures and the participant's stimulation. Immediately after stimulation offset, participants had to judge the experienced pleasantness or unpleasantness of stimulation by tapping on a rating scale on the touch screen (Fig. 1d), within maximally $3 \mathrm{~s}$ response time. The touched vertical screen coordinates were converted offline to a scale ranging from -10 over a neutral (0) midpoint to +10 . Participants either judged the emotions resulting from a trial for themselves (run "self judgment") or for the other participant (run "other judgment"). Crucially, the self-judgment condition controlled for general perceptual or cognitive confounds, such as visual and affective stimulus comparison, detection of incongruency, or overcoming general response conflict. In congruent trials, the two participants were touched by material of the same valence, whereas incongruent trials entailed unpleasant touch for one participant while the other was undergoing pleasant touch (and vice versa). Each run consisted of 40 pseudorandomized trials, with 20 pleasant (10 congruent/10 incongruent) and 20 unpleasant (10 congruent/10 incongruent) visuo-tactile stimuli. The stimuli had been chosen from a larger set of 40 stimuli used in a qualitative pretest in which a separate set of participants had been asked to evaluate the valence and congruency of the pictures and the materials combined. Those stimuli had been selected that elicited the highest agreement among participants in terms of emotional response and congruency, while at the same time inducing the strongest emotional responses (Table 1 shows the selected stimuli used in this study).

This experimental setup resulted in a three-factorial design (Fig. 2) with the factors "target" (self, other judgment), "valence" (pleasant, un- 
Table 1. List of stimuli

\begin{tabular}{lll}
\hline Pictures & Materials & Valence \\
\hline Puppy dog & Wool & Pleasant \\
Cotton plant & Cotton & Pleasant \\
Kitten & Synthetic fur & Pleasant \\
Brush & Soft Brush & Pleasant \\
Sheep & Wool & Pleasant \\
Rabbit & Synthetic fur & Pleasant \\
Swan & Feather & Pleasant \\
Cotton ball & Cotton & Pleasant \\
Rose & Silk & Pleasant \\
Chick & Feather & Pleasant \\
Mushroom 1 & Slimy mushroom & Unpleasant \\
Cow tongue & Softened Velcro & Unpleasant \\
Stinkbug & Plastic stinkbug & Unpleasant \\
Mushroom 2 & Gelatin & Unpleasant \\
Catfish & Small toy slime & Unpleasant \\
Maggots & Toy worms & Unpleasant \\
Liver & Toy slime & Unpleasant \\
Spider & Plastic spider & Unpleasant \\
Slug & Plastic slug & Unpleasant \\
Oyster & Toy slime & Unpleasant \\
\hline
\end{tabular}

pleasant stimulation), and "congruence" (congruent, incongruent stimulation of participant and other). Data were analyzed with corresponding ANOVAs and planned comparisons, performed using the IBM SPSS statistics software, version 20.0, and Statistica 7.0. We investigated the presence of a significant EEB by assessing for a specific interaction of congruency and target, that is whether the difference between incongruent versus congruent emotional judgments is higher for judgments about the other than for judgments about oneself (Fig. 3; computation of EEB).

\section{fMRI Experiment 1}

The aim of the first fMRI experiment was to assess the feasibility of the new paradigm in the scanner environment, and to explore the neural networks associated with EEB. This experiment essentially served as a pilot experiment, acting as an independent functional localizer for the second fMRI experiment. Eighteen participants were paired to a second participant previously unknown to them (in fact a female confederate of the experimenter) that was ostensibly performing the task outside the scanner room. Visual stimulus presentation was performed using a backprojection system consisting of a rear-view mirror mounted on the scanner's head coil. The experimenter sitting next to the scanning bed touched the left hand of the participant using the same materials as in the behavioral experiment. Each trial consisted of $10 \mathrm{~s}$ visuo-tactile stimulation, followed by emotion judgments on a Likert-type rating scale with nine discrete values (from -4 over 0 to +4 ). Ratings were entered by moving a cursor with right hand button presses using a response box. A longer stimulation time than in the behavioral experiment was chosen to allow exploring the time course of effects, using a finite impulse response (FIR) model. Ratings were rescaled off-line to a range from -10 over 0 to +10 (by multiplying each scale value by 2.5 ) to allow comparisons with the other experiments. Two scanning runs were performed (selfjudgment and other judgment), with order counterbalanced across participants. Trial number and order were identical to the behavioral experiment. A black fixation cross on white background was presented during intertrial intervals, which were jittered between 1000 and $2000 \mathrm{~ms}$ (mean intertrial interval [ITI] including a $4 \mathrm{~s}$ rating window $=5500 \mathrm{~ms}$ ).

MRI acquisition and analysis. A 3 Tesla Philips Achieva whole-body MR Scanner at the University Hospital Zurich, equipped with an 8-channel head coil, was used for MRI scanning. Structural images were acquired as $180 \mathrm{~T} 1$-weighted transversal images $(0.75 \mathrm{~mm}$ slice thickness). Functional images were acquired using a $\mathrm{T} 2{ }^{*}$-weighted echoplanar imaging (EPI) sequence with 33 transversal slices covering the whole brain (slice thickness $3.2 \mathrm{~mm}$; interslice gap $0.5 \mathrm{~mm}$; TR/TE $=$ $2000 / 35 \mathrm{~ms}$; flip angle $=82^{\circ}$, field of view $=220 \times 220 \mathrm{~mm}^{2}$; matrix size $=128 \times 128$, sense factor 2). A total of 385 volumes were obtained. Data were analyzed with SPM5 (Wellcome Department of Imaging Neu- roscience, London, UK). All functional volumes were realigned to the first volume, spatially normalized to the standard EPI template, and smoothed using a Gaussian kernel with full width at half maximum (FWHM) of $10 \mathrm{~mm}^{3}$. Following preprocessing, statistical analysis was performed using a general linear model approach. High-pass temporal filtering with a cutoff of $128 \mathrm{~s}$ was used to remove low-frequency drifts. Regressors of interest were convolved with the canonical hemodynamic response function. First-level regressors were modeled according to the target of emotion judgments, valence of stimulation, and congruency of valence. A separate regressor was defined for the rating window. The intertrial intervals (fixation) served as an implicit baseline. Residual effects of head motion were corrected by including the six estimated motion parameters of each participant as regressors of no interest in the design matrix. Exploration of the data with a FIR model showed significant activation restricted to the first seconds of a trial, whereas later regressors did not show any significant activation. Thus, following this initial assessment, the $10 \mathrm{~s}$ stimulation window was split into two regressors, one modeling early responses during the first $5 \mathrm{~s}$ of a trial and one regressor of no interest modeling the remaining $5 \mathrm{~s}$. Random effects group-level analyses were implemented using a full factorial ANOVA design with repeated-measures factors target (self and other), valence (pleasant and unpleasant), and congruency (congruent and incongruent). Linear contrasts of this ANOVA model were used to assess main effects and interactions. The main contrast of interest was the interaction contrast (Other Judgment Incongruent $>$ Congruent) $>$ (Self Judgment: Incongruent $>$ Congruent). Given the exploratory nature of this first experiment we performed whole-brain analyses with thresholds of $p$ values $<0.005$, uncorrected for multiple-comparisons, and a cluster size criterion of $k=10$ contiguous voxels. Such a rather liberal threshold was used to protect against false negatives in this first pilot investigation of EEB that mainly served to generate hypotheses about neural networks associated with it. Even at this liberal threshold, though, the only cluster revealed in the entire brain was in rSMG, and this cluster was subsequently used as a mask for the small-volume-correction analysis approach of fMRI Experiment 2 (see below).

PPI analyses. Psychophysiological interaction (PPI) analyses were performed according to the procedure described by Friston et al. $(1997,2002)$. This approach aims to determine whether the correlated activity of brain areas with a seed region is modulated by the psychological context, which in our case was the occurrence of incongruent (vs congruent) affective states. To this end, for each participant, the physiological activity of the seed region rSMG was defined as the first eigenvariate of the time series of all voxels within a $6 \mathrm{~mm}$ radius sphere, centered on the most significant voxel in rSMG identified by the interaction contrast of the random-effects analysis (rSMG: MNI 68/-38/32). The signal was deconvolved to estimate the underlying neuronal activity (Gitelman et al., 2003). The PPI regressors were obtained by multiplying the estimated (deconvolved) neural activity from the seed region with a vector coding for effects of incongruency (incongruent vs congruent) in the "other judgment" run. Then, on the first level, a whole brain analysis was performed using as predictor variables the PPI regressor, the experimental contrast (incongruent vs congruent), and the estimated neuronal activity from the seed region. Finally, individual PPI contrast images were entered into a random effects one-sample $t$ test to assess results on the group level. The clusters resulting from this analysis were subsequently used as the mask in the small-volume-correction analysis approach of fMRI Experiment 2.

\section{fMRI Experiment 2}

The aim of the second experiment was to establish the findings of the pilot fMRI Experiment 1 in a larger sample of 32 participants, and to assess how activation in rSMG associated with EEB was related to attention reorienting. The rationale for adding an attention reorienting task was based on the debate that attention reorienting could be a potential mechanism for explaining activation in rTPJ observed during self-other distinction required in "theory of mind" (Mitchell, 2008; Young et al., 2010).

Scanning runs and procedures were largely identical to fMRI Experiment 1 . However, the stimulation duration and response entry were now adapted to the procedures also used in the behavioral and the TMS experiment. More specifically, as fMRI Experiment 1 showed significant 


\section{Other Judgment}

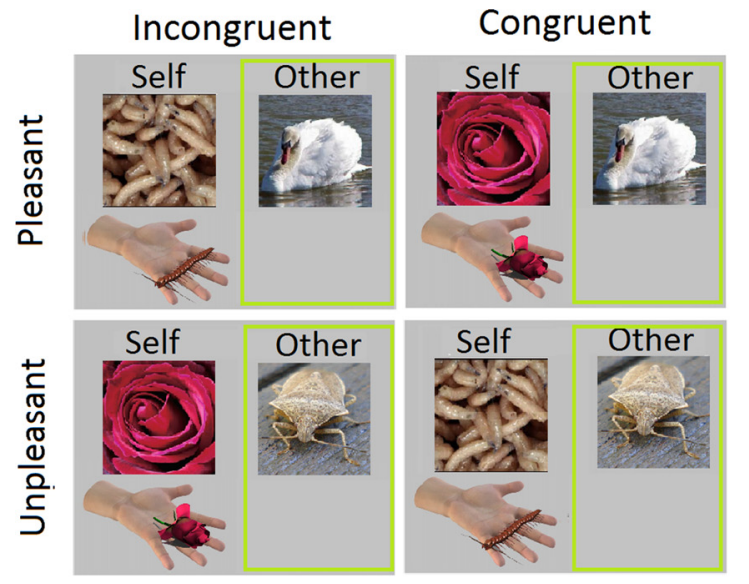

Self Judgment

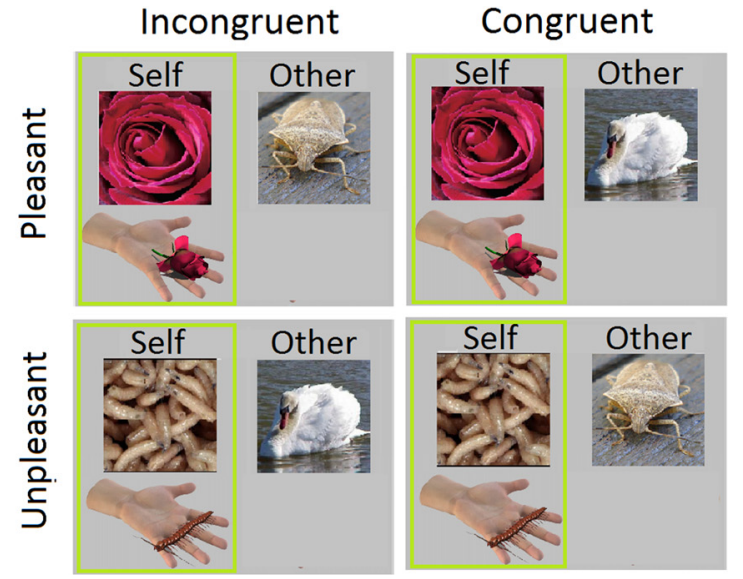

Figure 2. Schematic overview of the experimental design with the factors target (self/other judgment), valence (pleasant/unpleasant), and congruency (incongruent/congruent). The top rows of each gray box display what participants saw on the screen, and the bottom row is meant to illustrate concurrent touch. The green frame is shown to highlight the target (self or other) of the emotion judgment of each box for illustration purposes, but was actually not shown to participants.

\section{Behavioral ( $\mathrm{N}=72)$}

a

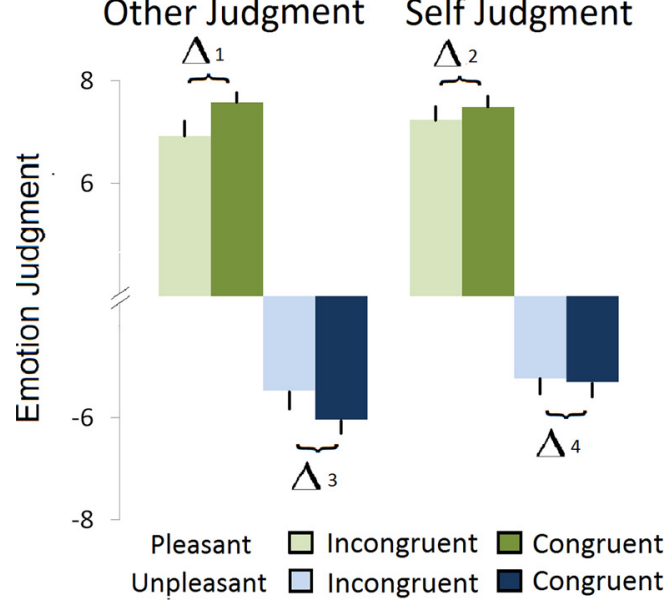

b

Emotional Egocentricity Bias

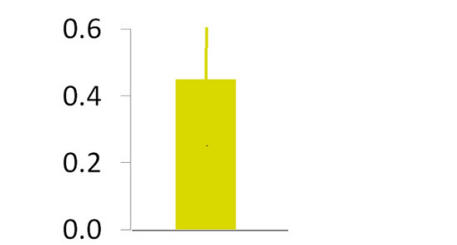

$\left[-1^{*}\left(\Lambda_{1}-\Lambda_{2}\right)+\left(\Lambda_{3}-\Delta_{4}\right)\right] / 2$

Figure 3. Behavioral ratings and EEB for the first behavioral experiment $(N=72)$. $\boldsymbol{a}$, Emotion judgments (mean $+\mathrm{SE}$ ) are plotted for each condition of the factorial design. $\boldsymbol{b}$, EEB (mean $+\mathrm{SE}$ ) is pooled across pleasant and unpleasant judgments. For descriptive purposes, EEB was explicitly calculated by subtracting other-related emotion judgments (run "other judgment") during congruent trials from other-related judgments during incongruent trials. From this, as a control, we subtracted the differences between congruent and incongruent trials in the "self judgment" run.

responses only within the first seconds of stimulation, stimulation time was shortened to $3 \mathrm{~s}$, as in the behavioral and TMS experiment. In addition, ratings were entered using an MRI-compatible track ball moving a cursor on the same visual analog scale as the one used in the behavioral and TMS experiment (with response times also restricted to $3 \mathrm{~s}$, and with scale values also converted to range from -10 over 0 to +10 ).

MRI acquisition and analysis. fMRI scanning was performed at the Laboratory for Social and Neural Systems Research, Zurich, on a 3 Tesla Philips Achieva whole-body MR Scanner equipped with an 8-channel head coil. Structural images were acquired as $180 \mathrm{~T} 1$-weighted transversal images $(0.75$ $\mathrm{mm}$ slice thickness). Twenty-six transversal slices covering the whole brain with a slice thickness of $3.5 \mathrm{~mm}$ were acquired (interslice gap of $0.5 \mathrm{~mm}$; $\mathrm{TR} / \mathrm{TE}=1500 / 35 \mathrm{~ms}$; flip angle $=82^{\circ}$, field of view $=220 \times 220 \mathrm{~mm}^{2}$; matrix size $=80 \times 80$, sense factor 2$)$. A total of 345 volumes were scanned for each run. Data were analyzed with SPM5 (Wellcome Department of Imaging Neuroscience, London, UK). Preprocessing and statistical analyses were performed in the same fashion as in fMRI1, but with only one eventrelated regressor covering the shorter stimulation time. Group-level statisti- cal inference was based on the small volume correction approach (SVC). This entailed using the results of each corresponding contrast obtained in the previously performed fMRI Experiment 1, therefore defining a restricted a priori search space (volume of interest, VOI) from a strictly independent sample. Activations were thresholded using multiple-comparison correction based on random Gaussian field theory within this search space, at a threshold of $\mathrm{P}(\mathrm{SVC})=0.05, k=10$. This represents a double-tiered strategy where hypotheses generated by the first, exploratory experiment (fMRI1) were tested with more strict control of false positives using fMRI Experiment 2 (Poldrack, 2007). Importantly, the data from fMRI1 and fMRI2 were not quantitatively combined in a joint analysis, but treated as two independent observations. Data from the exploratory study fMRI1 were thus chosen as fully independent "localizers" for region-of-interest statistical inference in fMRI2. This approach was also preferred to a direct quantitative comparison because the data had been acquired on two different scanners, with different sample sizes and slightly different procedures (stimulation time, response format, and device; Kriegeskorte et al., 2010 shows how this approach follows recommendations about independence of analyses). In addition, to 
a

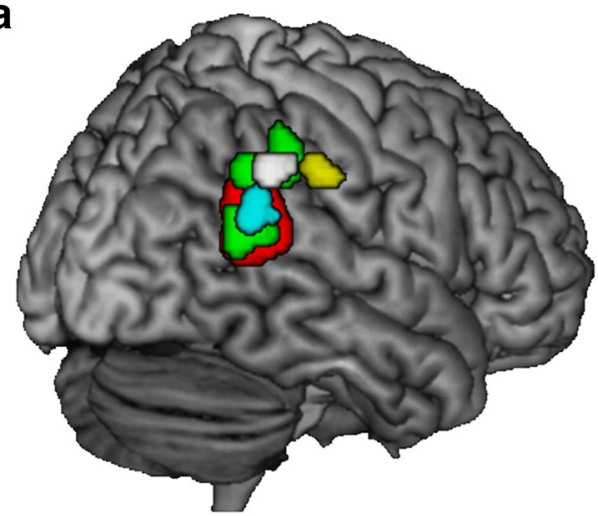

fMRI EEB
fMRI attention reorienting
Imitation-inhibition tasks
Agency tasks
Theory of Mind tasks

b

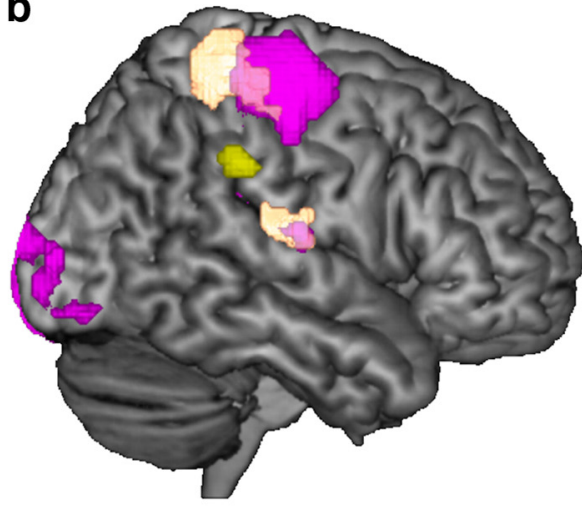

O Seed region: rSMG

Increased connectivity with rSMG

$\mathrm{O}$ SI \& SII hand area

Figure 4. Results of the second fMRI experiment. $\boldsymbol{a}$, Comparison of neural response in rSMG related to EEB as identified by the interaction contrast of the random-effects analysis (yellow) to reorienting of attention to task-relevant stimuli (white), theory of mind (red; Mar, 2011), imitation inhibition (turquoise; Brass et al., 2005, 2009; Spengler et al., 2009), and agency (green; Sperduti et al., 2011). $\boldsymbol{b}$, Results of the PPI analysis. Increased connectivity for incongruent vs. congruent trials during the other-related judgments with the seed region rSMG (yellow) is depicted in violet. Activations in light pink show areas in primary and secondary somatosensory cortex responding to stimulation of the left hand (Eickhoff et al., 2008).

explore activation outside the VOIs, we performed a whole-brain analysis ( $p=0.05$, with random Gaussian field multiple-comparison correction). The Anatomy Toolbox (version 1.6; Eickhoff et al., 2005) and an anatomical atlas (Duvernoy, 1991) were used for anatomical and cytoarchitectonic interpretation. The PPI analyses were performed in the same fashion as in fMRI1, with the seed region based on the peak of the interaction contrast in fMRI Experiment 2 (rSMG: MNI 68/-38/36).

Additional paradigm: attention reorienting task. The attention reorienting task was set up exactly as in the "attentional cueing" paradigm used by Mitchell (2008), which had been derived from the spatial cueing paradigm of Posner et al. (2007). Participants were asked to indicate, by pressing with their index or middle finger on a response-box, the location of a visual target stimulus that appeared on the left or right side of the screen. The location of the visual target was congruent or incongruent to the direction of a horizontal arrow pointing to the left or to the right, immediately preceding the target appearance on the screen. In each trial, subjects were presented with a fixation cross on the center of the screen and two white squares at either side. After $700 \mathrm{~ms}$, the horizontal line of the cross was replaced with an arrow pointing to either the left or right square. After a variable interstimulus interval (ISI 500-2000 ms), a white asterisk appeared in either the left or right box $(100 \mathrm{~ms})$ and participants were asked to respond as fast as possible by pressing the button corresponding to the location of the visual target. Correct responses and reaction time were recorded for each subject. On $75 \%$ of the trials, the target appeared in the square to which the arrow had pointed (congruent trials), whereas on the remaining $25 \%$ of the trials, the target appeared in the square opposite to the side where the arrow had pointed (incongruent trials). Participants completed a total of 160 trials (120 valid, 40 invalid), which were separated into two runs of $512 \mathrm{~s}$ each. A total of 700 volumes were obtained. MRI scanning acquisition and preprocessing procedure were identical to the other runs of fMRI Experiment 2. Separate regressors coding for congruent and incongruent trials were defined (Mitchell, 2008). Random effects group analyses were implemented using a paired two-sample $t$ test on those regressors. Linear contrasts were used to assess differences between incongruent and congruent trials. Results were small volume corrected $[\mathrm{P}(\mathrm{SVC})=0.05]$ for a rTPJ volume of interest defined by a meta-analysis exploring attention orienting as a mechanism for self- other distinction (Decety and Lamm, 2007). To as- sess the overlap of this activation with the rSMG activation related to $\mathrm{EEB}$, we performed a random effects conjunction analysis (reorienting: incongruent $>$ congruent $\cap$ EEB interaction contrast), and complemented it by direct comparisons of the contrasts of interest (EEB interaction contrast vs reorienting: incongruent $>$ congruent).

Meta-analyses of theory of mind, agency, and imitation inhibition Classical theory of mind tasks, such as the Sally and Anne task, require self-other distinction as the observer needs to disentangle his or her own privileged knowledge about a scenario from what another, more naive person knows. This has been shown to recruit a distributed set of brain areas, including the rTPJ. In the motor domain, when interacting with others, an actor needs to keep track whether a movement originated in himself or herself, or in another actor. Hence, studies on agency as well as on inhibiting imitative tendencies have revealed that rTPJ as well as prefrontal areas play an important role in disentangling one's own motor representations from those of others. Therefore, to compare our results to previous findings on self-other distinction in the cognitive and motor domain, we quantitatively compared the results from our second fMRI experiment to meta-analyses of activations related to theory of mind, agency, and imitation inhibition. Meta-analytic activation in rTPJ during theory of mind was provided by Raymond A. Mar, and consisted of a joint analysis of story-based and nonstory based theory of mind studies collected in his coordinate-based meta-analysis (Mar, 2011). Results of this analysis were provided as a NIfTI File thresholded at $p=0.01$, FDR-corrected. Results of the coordinate-based meta-analysis for agency were provided by Sperduti (Sperduti et al., 2011), and included the contrast "other agency" of that paper, thresholded at $p=0.05$, FDRcorrected. As for the imitation inhibition studies, the low number of studies precluded a formal meta-analysis. We therefore converted the peak coordinates of the available three papers (Brass et al., 2005, 2009; Spengler et al., 2009) to MNI space, and smoothed them with a Gaussian kernel of $6 \mathrm{~mm}$ FWHM for display purposes (Fig. 4a). Activation related to these tasks and to EEB was compared by visualizing the activation clusters of the meta-analyses in standard stereotactic space together with activation related to EEB. 


\section{TMS experiment}

The goal of the TMS experiment was to investigate how the ability to minimize EEB can be interfered with. To this end we aimed to determine whether the specific role of rSMG as identified by the fMRI findings was to instantiate or, alternatively, to overcome egocentricity. The sample of the TMS experiment consisted of 45 right-handed female participants, 22 of which underwent rTMS of rSMG, and 23 of a neutral control region (the vertex). Stimulation sites were determined using individual structural scans and BRAINSIGHT frameless stereotaxy (Rogue Research). The vertex site was defined as the meeting point of the two central sulci in the intrahemispheric fissure. Vertex stimulation was used as a control for the nonspecific effects of TMS, such as auditory and sensory stimulation due to clicking sounds and tapping sensations, with minimal neural stimulation (as the interhemispheric fissure was the stimulation spot). Because fMRI2 and rTMS studies were run in parallel, the rSMG site was determined as the activation peak from the interaction contrast in fMRI1 (note, however, that the activation peaks in fMRI1 and fMRI2 were practically identical (fMRI1 MNI $x / y / z=68 /-38 / 32$ vs fMRI2 68/-38/36). For each participant, the rSMG peak was transformed into her structural scan's native space by means of the parameter estimates for spatial normalization generated by the segmentation of the anatomical image in SPM5. The TMS coil was positioned over these sites tangentially to the cortical surface (with the handle pointing posterior for vertex TMS, and 45 degrees coronal-to-posterior for rSMG TMS), and held in place by means of a mechanical holder. rTMS was applied at $1 \mathrm{~Hz}$ for $15 \mathrm{~min}$, immediately followed by an experimental run (either "self judgment" or "other judgment" order counterbalanced across participants) that lasted $\sim 7 \mathrm{~min}$. The full run thus fell well within the reported persistence of $1 \mathrm{~Hz}$ rTMS effects, reported to last half of the stimulation time (Eisenegger et al., 2008). The number of trials and the experimental procedures were identical to the behavioral experiment. Stimulation intensity was set to $110 \%$ of individually determined resting motor threshold [RMT; $\mathrm{M}(\mathrm{SE})=61.2 \%( \pm 1.3) \%$ total output], with RMTs determined before rTMS as the minimum intensity that produced visible twitches in the first dorsal interosseus in 3 of 5 trials for single-pulse TMS over the hand representation in right primary motor cortex. A between-subjects design was preferred over a within-subjects design, as the latter would have required participants to perform the experiment twice. This could have resulted in confounding effects of habituation and practice; moreover, participants would have had to undergo TMS stimulation four times (twice of the vertex, for independent "self" and "other" judgment runs, and twice of rSMG).

\section{Behavioral Experiment 2}

As a last step in our multimethod integrative approach we performed another behavioral experiment. This experiment aimed to replicate the EEB findings and additionally explored whether the ability to overcome EEB could be reduced by shortening the duration of visuo-tactile stimulation, and hence the time provided to regulate potentially biased judgments. This additional experiment was motivated by the observation that although our novel paradigm could establish the existence of EEB in the first behavioral experiment, participants seemed to be very proficient in overcoming EEB, as indicated by an effect size estimate $\left(\eta_{\mathrm{p}}^{2}=0.074\right)$ which by statistical conventions would be considered as medium, with values of $\eta_{\mathrm{p}}^{2}=0.01, \eta_{\mathrm{p}}^{2}=0.06$, and $\eta_{\mathrm{p}}^{2}=0.14$ representing small, medium, and large effects, respectively (Kirk, 1996). Note though that this is a statistical and not a conceptual classification, and that the impact of EEB will depend on the context and the possible implications of an impeded social interaction, and that even small effect sizes can result in substantial and highly relevant behavioral changes (Rosenthal and Rubin, 1982). However, although a medium behavioral effect size is in itself not problematic for neuroscientific investigations of processes involved in controlling this bias, it restricts the utility of our paradigm for purely behavioral investigations, especially when only small samples are available such as when assessing the degree of emotional egocentricity in clinical or developmental populations. The rationale for reducing visuotactile stimulation time was motivated by the fMRI results which had indicated that early perceptual integration processes in rSMG might help us to regulate egocentric responses arising at the beginning of stimulation (see Results and Discussion). Hence, we assumed that egocentricity effects are stronger at the onset of stimulation, and that shorter stimulation times might make it easier to detect such effects behaviorally, as the control processes involved in overcoming them might not have been engaged yet. Therefore, we used exactly the same procedures and stimuli as in the first behavioral experiment, except for reducing stimulation time to $1 \mathrm{~s}$.

\section{Results}

\section{Emotional egocentricity bias: behavioral Experiment 1}

A three-factorial ANOVA of the rating data was used to assess EEB in the first behavioral experiment $(N=72)$. We observed a significant interaction target $\times$ congruency $\left(F_{(1,71)}=5.659, p=0.02, \eta_{\mathrm{p}}^{2}=\right.$ 0.074). Inspection of the pattern of results (Fig. 3) and planned linear comparisons indicated that this interaction was driven by a significantly larger difference between incongruent and congruent otherrelated judgments [target other: incongruent - congruent $($ mean $/ \mathrm{SE})=0.616 / 0.211$, planned comparison of difference: $F_{(1,71)}=8.534, p=0.005$ ] than for self-related judgments [target self: incongruent - congruent $=0.165 / .115$, planned comparison; $F_{(1,71)}=2.052, p=0.156$; see also the plot and the explicit computation of EEB in Fig. 3]. In addition, the absence of a three-way interaction with valence $(F<1)$ documents that EEB is of similar size for positive and negative emotions (note that for all the ANOVAs, the sign of emotion judgments related to unpleasant stimulations has been reverted. This was done to ensure that all ratings always carried the same sign).

In addition to these main findings, the ANOVA showed significant main effects of the factors "valence" $\left(F_{(1,71)}=50.976\right.$, $\left.p<0.001, \eta_{\mathrm{p}}^{2}=0.418\right)$, and "congruency" $\left(F_{(1,71)}=7.668, p=\right.$ $\left.0.007, \eta_{\mathrm{p}}^{2}=0.097\right)$. All the other main effects and interactions were not significant (target, $F_{(1,71)}=2.328, p=0.132, \eta_{\mathrm{p}}^{2}=$ 0.032 ; target $\times$ valence, $F_{(1,71)}=2.644, p=0.108, \eta_{\mathrm{p}}=0.036$; congruency $\times$ valence, $F_{(1,71)}=2.388, p=0.127, \eta_{\mathrm{p}}^{2}=0.033$; target $\times$ valence $\times$ congruency, $\left.F_{(1,71)}<1\right)$. The main effect of congruency resulted from higher values in the congruent trials [congruent $($ mean $/ \mathrm{SEM})=6.570 / .177$; incongruent $=6.180 /$ .222], and the main effect of valence signified higher intensity ratings for pleasant than for unpleasant stimulation (pleasant $=$ 7.234/.198, unpleasant $=5.515 / .246$ ).

\section{Brain areas subserving EEB assessed by fMRI}

ANOVAs of the behavioral data acquired in both $\mathrm{fMRI}$ experiments revealed that using the paradigm in the scanner environment resulted in generally similar types of responses as in the first behavioral experiment, in particular regarding overall valence judgments. Although the effects pointed in the same direction, however, the behavioral data did not reach significance for EEB (fMRI1: target $\times$ congruency, $F_{(1,17)}=1.233, p=0.282, \eta_{\mathrm{p}}^{2}=0.068$, mean EEB $=$ 0.264 ; fMRI2: target $\times$ congruency, $F_{(1,31)}=0.235, p=0.631, \eta_{\mathrm{p}}^{2}=$ 0.008 , mean $\mathrm{EEB}=0.085$ ).

To assess the brain regions involved in EEB, we computed the contrast (other judgment: incongruent $>$ congruent) $>$ (self judgment: incongruent $>$ congruent). Recall that all results reported here are from $\mathrm{AMRI}$, with search volumes restricted by the clusters obtained from the fully independent pilot experiment fMRI1 in the same contrasts. A significant cluster was revealed in posterior rSMG (Fig. $4 a$, yellow cluster; MNI coordinates $x / y / z=$ $68 /-38 / 36, \mathrm{P}_{\mathrm{SVC}}=0.036$; Table 2), whereas the opposite contrast (self judgment: incongruent $>$ congruent $)>$ (other judgment: incongruent $>$ congruent) did not show any significant voxels. In line with the behavioral data, the three-way interaction target $X$ congruency $\times$ valence also did not yield significant activations, nor did the additionally performed whole-brain analysis testing for activations outside the ROI. 
Table 2. fMRI experiment 2

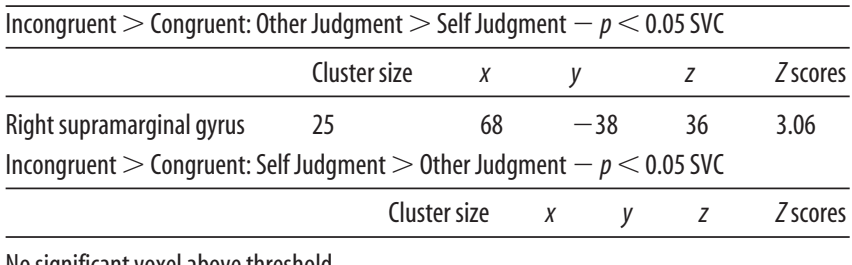

No significant voxel above threshold

Table 3. fMRI experiment 2

\begin{tabular}{lcccrc}
\hline Increased connectivity 0 ther Judgment: Incongruent $>$ Congruent $-p<0.05$ SVC \\
\hline \multicolumn{7}{c}{ Cluster size } & $x$ & $y$ & $z$ & Zscores \\
\hline Right postcentral gyrus & 39 & 44 & -26 & 64 & 4.16 \\
Right rolandic operculum & 97 & 54 & -16 & 14 & 4.12 \\
Left inferior occipital gyrus & 41 & -36 & -78 & -12 & 4.64 \\
Decreased connectivity $-p$ & $<0.05$ SVC & & & & \\
\hline & Cluster size & $x$ & $y$ & $z$ & Z scores \\
\hline
\end{tabular}

No significant voxel

PPI analysis with rSMG (68/-38/36) as the seed region.

Hence, the first and second fMRI experiment consistently showed higher activity in rSMG, and only in rSMG, when participants had to make empathic judgments about another persons' affective states (but not when engaged in self-judgments) when they were in an incongruent as compared with a congruent emotional state. Note that as in the behavioral experiment, these activations cannot be attributed to general confounds related to incongruency detection or conflict resolution, as these processes were cancelled out by subtracting the self-judgment incongruency contrast.

The results of the psychophysiological interaction analysis further revealed that the cluster in rSMG showed increased effective connectivity (Fig. $4 b$; Table 3 ) for incongruent versus congruent trials with primary (SI) and secondary (SII) somatosensory cortex, notably contralateral to the stimulated hand, and with bilateral higher-order visual cortex (SI, 44/-26/64, Psvc $=$ 0.001 ; SII, 54/-16/14, Psvc $=0.004$; visual $-36 /-78 /-12$, $\mathrm{P}_{\mathrm{SVC}}=0.01$; SVC based on PPI results from pilot experiment fMRI1). This increased interaction of rSMG with both visual and somatosensory brain areas suggests a possible mechanism to resolve incongruent incoming information related to the self (somatosensation) versus the other (vision).

\section{Functional segregation of rTPJ and rSMG assessed by fMRI and meta-analyses}

Previous social neuroscience research has consistently implicated the rTPJ in theory of mind and self-other distinction tasks (Decety and Lamm, 2007; Corbetta et al., 2008; Brass et al., 2009; Mar, 2011), as well as in attention reorienting acting as a possible mechanism for self-other distinction (Mitchell, 2008; Young et al., 2010). However, we reliably observed activation in the rSMG associated with EEB in a location that was clearly anterior to the commonly observed rTPJ activity for social-cognitive and attention paradigms (i.e., in the junction between anterior inferior parietal lobule, posterior superior temporal, and angular gyrus). To directly test for this possible functional segregation between EEB-related activation in rSMG and rTPJ activation reported in previous research, we performed two additional analyses. First, using a within-subject comparison, the activation in rSMG was compared with activation related to attention reorienting, as de- termined by means of the spatial cueing paradigm of fMRI Experiment 2 . In line with other studies and meta-analyses (Decety and Lamm, 2007; Corbetta et al., 2008; Mitchell, 2008; Scholz et al., 2009), reorienting of attention revealed significant activation in rTPJ (peak coordinates $60 /-48 / 27, \mathrm{P}_{\text {SVC }}=0.016$ ). This activation cluster was clearly distinct from the rSMG activation measured in the same participants, without any overlapping voxels (even when lowering the threshold to a very liberal $p=0.01$, uncorrected for multiple-comparisons; Fig. $4 a$, white outline). However, direct comparison of activation strengths in the two tasks (by means of statistical contrasts) only revealed a trend toward significantly higher activation for attention reorienting in the more posterior part of $\mathrm{rTPJ}[60 /-56 / 36, \mathrm{P}$ (uncorr) $=0.003]$. Second, the comparison of activation in rSMG to meta-analyses of rTPJ during theory of mind (Mar, 2011), agency (Sperduti et al., 2011), and imitation inhibition (Brass et al., 2009) did not yield any overlapping voxels.

\section{Increasing EEB: the causal role of rSMG assessed by the TMS experiment}

A central question of our study was to identify the mechanisms by which biased judgments can be avoided. The fMRI and behavioral results reported so far indicate that (1) empathic judgments are biased toward the participant's own emotions when they are incongruent to those of the other person; (2) that the effect size of EEB is medium, indicating that participants are generally rather proficient in minimizing biased judgments; and (3) that biased judgments are accompanied by increased neural processing in the rSMG. Due to the correlative nature of fMRI, however, these findings cannot disclose whether activity in this area instantiates the bias, or whether it is related to ensuring objective judgments by overcoming egocentricity. We tested these opposing hypotheses by temporarily disrupting rSMG functionality using $1 \mathrm{~Hz}$ rTMS, which is generally thought to inhibit cortical activity (Rossi et al., 2009). If such disruptive stimulation were to result in a reduction of EEB, this would speak for the role of $\mathrm{rSMG}$ in causing biased judgments. If, to the contrary, rTMS of rSMG were to result in an increase of EEB, this would indicate that this area is involved in overcoming biased judgments.

Analysis of the rTMS experiment with a mixed-model ANOVA with the between-subjects factor group (rSMG vs vertex) and the within-subjects factors target (other vs self), valence (pleasant vs unpleasant), and congruency (congruent vs incongruent) revealed a significant three-way interaction group $\times$ target $X$ congruency. Inspection of the pattern of results (Fig. 5) and planned linear comparisons indicated that this interaction was driven by a significantly larger difference between incongruent and congruent other-related judgments for the rSMG group than for the vertex control group, whereas no difference between the groups was observed for self-related judgments (rSMG: other: incongruent - congruent $=1.489 / .500$, self: incongruent - congruent $=0.285 / .172$; vertex: other: incongruent - congruent $=$ $0.232 / .264$; self: incongruent - congruent $=0.157 / .101$; planned comparison rSMG vs vertex target other, $F_{(1,47)}=5.069, p=$ 0.03 ; target self, $F<1$ ). The mean size of EEB was $\sim 3$ times as high as in the first behavioral experiment (1.204 vs 0.451 scale units), and showed a $2.4 \%$ increase of explained variance (from $\eta_{\mathrm{p}}^{2}=0.074$ to $\eta_{\mathrm{p}}^{2}=0.098$, corresponding to a relative increase of $\sim 32 \%$ ). Additional statistical tests aimed to determine whether the significant effects in the TMS experiment could indeed be attributed to TMS of rSMG increasing the bias, as opposed to TMS of the vertex decreasing it. Because of the different sample sizes and variance structures in the TMS and 


\section{rTMS: right SMG $(\mathrm{N}=22)$}

a

Other Judgment Self Judgment

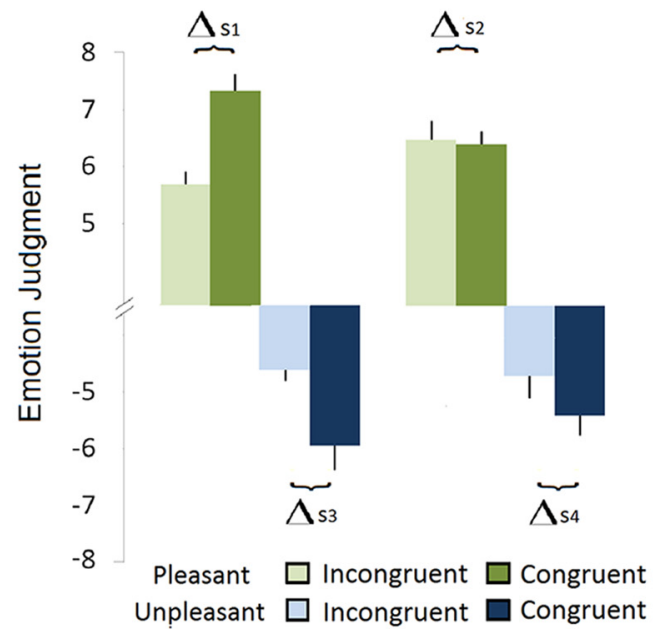

b

\section{Emotional Egocentricity Bias}

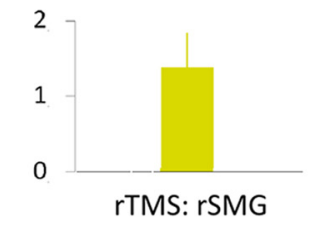

$\left[-1^{*}\left(\Lambda_{s 1}-\Lambda_{s 2}\right)+\left(\Lambda_{s 3}-\Lambda_{s 4}\right)\right] / 2$

\section{rTMS: Vertex $(\mathrm{N}=23)$}

\section{Other Judgment Self Judgment}

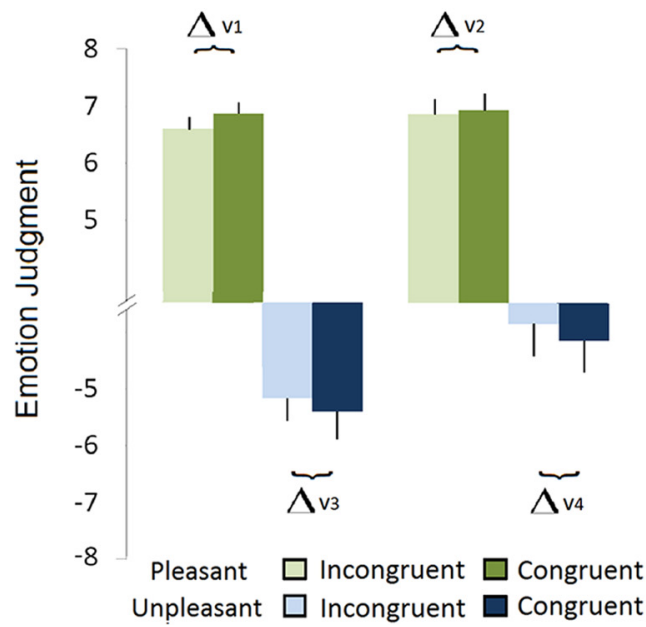

d Emotional Egocentricity Bias

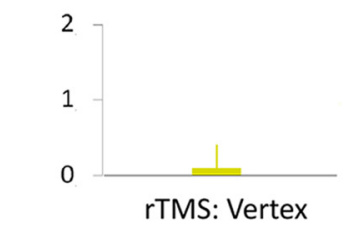

$\left[-1^{*}\left(\Delta_{\mathrm{v}_{1}}-\Delta_{\mathrm{v}_{2}}\right)+\left(\Delta_{\mathrm{v}}-\Delta_{\mathrm{v}_{4}}\right)\right] / 2$

Figure 5. Behavioral ratings and EEB for the rTMS experiment. $\boldsymbol{a}, \boldsymbol{c}$, Emotion judgments (mean $+\mathrm{SE}$ ) for the rSMG and the vertex control groups are plotted separately for each condition of the factorial design $(\boldsymbol{b}, \boldsymbol{d}) \mathrm{EEB}$ (mean $+\mathrm{SE}$ ) in the two groups (averaged across EEB in the pleasant and unpleasant domain). Note that the scale range as compared with EEB in the first behavioral experiment depicted in Figure 3 is much larger, as TMS on rSMG resulted in a threefold increase of EEB.

the behavioral experiment, a directed independent sample nonparametric Mann-Whitney $U$ test was performed which revealed that EEB in the rSMG group showed a significant increase $\left(\mathrm{P}_{\mathrm{U}}=0.034\right)$, whereas EEB in the vertex group did not significantly differ from EEB in the behavioral experiment $\left(\mathrm{P}_{\mathrm{U}}=0.23\right)$. These results suggest that rSMG is causally involved in overcoming EEB, as EEB increased when disrupting rSMG functioning by rTMS.

In terms of further findings, the ANOVA also revealed a significant main effect of valence $\left(F_{(1,43)}=25.612, p<0.001\right.$, $\left.\eta_{\mathrm{p}}^{2}=0.373\right)$ and of congruency $\left(F_{(1,43)}=10.962, p=0.002\right.$, $\left.\eta_{\mathrm{p}}^{2}=0.203\right)$. Significant interactions were found for group $\times$ congruency $\left(F_{(1,43)}=4.486, p=0.04, \eta_{\mathrm{p}}^{2}=0.094\right)$, target $\times$ valence $\left(F_{(1,43)}=5.702, p=0.021, \eta_{\mathrm{p}}^{2}=0.117\right)$, target $\times$ congruency $\left(F_{(1,43)}=5.967, p=0.019, \eta_{\mathrm{p}}^{2}=0.122\right)$, group $\times$ target $\times$ congruency $\left(F_{(1,43)}=4.649, p=0.037, \eta_{\mathrm{p}}^{2}=0.098\right)$, and target $\times$ valence $\times$ congruency $\left(F_{(1,43)}=4.848, p=0.033\right.$, $\left.\eta_{\mathrm{p}}^{2}=0.101\right)$. All other terms were not significant, including group $\times$ target $\times$ congruency $\times$ valence $(p=0.168)$, suggesting that TMS effects were similar in both the pleasant and unpleasant domain.

\section{Increasing EEB by reducing stimulation time: behavioral}

\section{Experiment 2}

The ANOVA of the second behavioral experiment with reduced stimulation duration showed a significant interaction target $X$ 


\section{Behavioral $(\mathrm{N}=24)$}

a

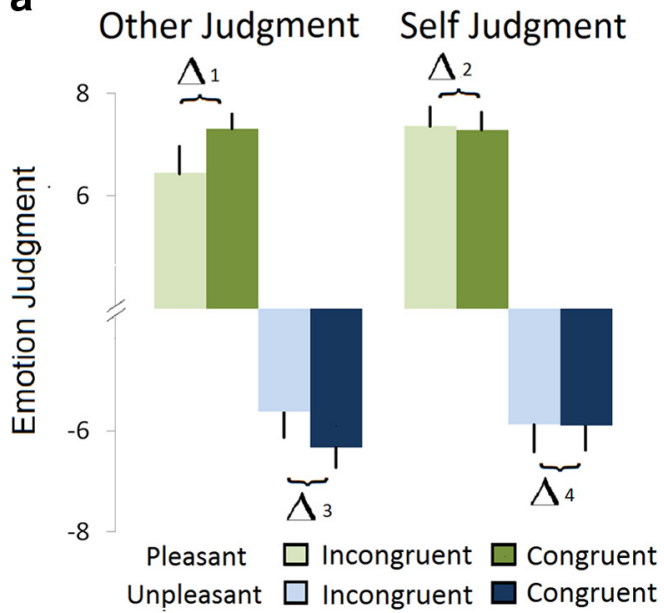

b

Emotional Egocentricity Bias

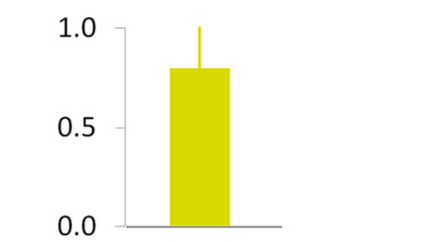

$\left[-1^{*}\left(\Lambda_{1}-\Lambda_{2}\right)+\left(\Lambda_{3}-\Lambda_{4}\right)\right] / 2$

Figure 6. Behavioral ratings and EEB for the second behavioral experiment with shorter stimulation time $(N=24)$. As in Figure 3. $\boldsymbol{a}$, Emotion judgments (mean $+S E)$ are plotted for each condition of the factorial design. $\boldsymbol{b}$, EEB (mean $+\mathrm{SE}$ ) is pooled across pleasant and unpleasant judgments. For descriptive purposes, EEB was explicitly calculated by subtracting other-related emotion judgments (run "other judgment") during congruent trials from other-related judgments during incongruent trials. From this, as a control, we subtracted the differences between congruent and incongruent trials in the "self judgment" run.

congruency $\left(F_{(1,23)}=8.832, p=0.007, \eta_{\mathrm{p}}^{2}=0.277\right)$. Inspection of the pattern of results (Fig. 6) and planned linear comparisons indicated again that this interaction was driven by a significantly larger difference between incongruent and congruent otherrelated judgments $\left(0.774 / .281\right.$; planned comparison, $F_{(1,23)}=$ 7.544, $p=0.011)$ than self-related judgments $(-0.025 / .005$; planned comparison, $\left.F_{(1,23)}<1\right)$. Thus, as expected, shortening stimulation time and hence the time for regulatory control diminished the ability to overcome egocentric judgments, and substantially increased the effect size of EEB as documented by a $\eta_{\mathrm{p}}^{2}$ that was considerably higher than in the first behavioral and the TMS experiment ( 0.277 vs 0.074 and 0.098 , respectively). Notably, EEB in the second behavioral experiment was significantly higher than in the first experiment (Mann-Whitney $U$ test, $\mathrm{P}_{\mathrm{U}}=$ 0.02 ), but not significantly higher than in the rSMG TMS group $\left(\mathrm{P}_{\mathrm{U}}=0.676\right)$.

All other effects were largely identical to those of the first experiment (significant effects for valence $\left(F_{(1,23)}=9.686, p=\right.$ $\left.0.005, \eta_{\mathrm{p}}^{2}=0.296\right)$, and congruency $\left(F_{(1,23)}=4.884, p=0.037\right.$, $\eta_{\mathrm{p}}^{2}=0.175$; nonsignificant effects for target, $F_{(1,23)}=1.130, p=$ $0.299, \eta_{\mathrm{p}}^{2}=0.047$; target $\times$ valence, $F_{(1,23)}=2.542, p=0.125$, $\eta_{\mathrm{p}}^{2}=0.100$; congruency $\times$ valence, $F_{(1,23)}<1$; target $\times$ valence $\times$ congruency, $\left.F_{(1,23)}<1\right)$. The results of this behavioral experiment with shorter stimulation time therefore demonstrate that (1) we could replicate the existence of EEB as demonstrated already in Experiment 1, and more importantly, (2) we could show that the reduction of the stimulation duration from $3 \mathrm{~s}$ to $1 \mathrm{~s}$ leads to a remarkable increase in the size of the EEB, allowing its detection in a small sample and by consequence providing a useful behavioral paradigm for testing emotional egocentricity in patient populations.

\section{Discussion}

In the last years, social cognitive neuroscience has identified neural activations underlying the first-person experience of emotions as a potential neural mechanism subserving the empathic understanding of others. Here, we draw attention to the fact that such a simple self-projection mechanism can also lead to egocen- trically biased judgments and therefore needs to be complemented by an additional mechanism enabling us to overcome them in situations when relying on information from one's own emotional state would be misleading. Based on a newly developed paradigm that induces emotions by means of visuo-tactile stimulation, we could successfully detect the existence of such an EEB in healthy adults and quantify the degree to which human adults exert it. Furthermore, we identified a neuronal mechanism that enables us to overcome such emotional egocentricity and the conditions under which such biased judgments increase.

The results of our first series of experiments suggest that under normal circumstances, healthy adults are rather proficient in overcoming egocentric response tendencies. In an initial experiment with 72 subjects, we observed a significant EEB with medium effect size. EEB could however be drastically increased when either disrupting the neuronal mechanisms necessary to overcome egocentric judgments, using TMS, or when reducing the time given to participants to disentangle their own and the other's emotions.

The ability to distinguish self- from other-related representations is a prime function of social cognition in general, and of empathy in particular (Singer and Lamm, 2009). Self-other distinction has often been reported to relate to neural activity either in the rTPJ (Morishima et al., 2012; Santiesteban et al., 2012), or in medial prefrontal areas. For instance, it has been demonstrated that mentalizing about others who have either similar or dissimilar attitudes than the self engages distinct areas in dorsal and ventral medial prefrontal cortex, respectively (Mitchell et al., 2006). Conceptually related findings were reported by Spengler et al. ( 2009), who showed that anterior medial prefrontal cortex as well as rTPJ were engaged when overcoming the interfering effects of other people's motor intentions on one's own incongruent motor intentions, as opposed to congruent intentions. Recently, it has also been shown that making empathic choices requires simulation and self-other distinction operating at the same time, with activity in prefrontal and inferior parietal cortices coding for these two processes, respectively (Janowski et al., 2013; Nicolle et al., 2012). Notably, the activations in IPC as reported by Janowski et al. (2013) were in close 
Table 4. MNI coordinates and Euclidean distance between peak coordinates of rSMG activation related to EEB, and peak coordinates in rTPJ from meta-analyses of imitation-inhibition, agency, theory of mind, and the attention reorienting task performed in the same participants

\begin{tabular}{llllllllc}
\hline Study & $x$ & $y$ & $z$ & 1 & 2 & 3 & 4 & 5 \\
\hline (1) fMRI 2 & 68 & -38 & 36 & & 15.1 & 22.4 & 25.5 & 15.7 \\
(2) Imitation-Inhibition & 60 & -48 & 28 & & & 8.1 & 10.8 & 1 \\
(3) Agency & 53 & -52 & 27 & & & & 5.1 & 8.1 \\
(4) Theory of Mind & 52 & -52 & 22 & & & & & 10.2 \\
(5) Attention reorienting & 60 & -48 & 27 & & & & & \\
\hline
\end{tabular}

vicinity to the rSMG area associated with EEB. Together, these findings suggest that simulation mechanisms might be at play when we infer the internal states of others, but that an additional mechanism is required to disengage from simulation in cases where it would not be appropriate.

Interestingly, the results of our two fMRI experiments consistently show that self-other distinction in the EEB paradigm engages an area anterior to what has been previously referred to as rTPJ. This area was located in the SMG and thus anterior to the junction of parietal and temporal cortex. Functional segregation between rTPJ "proper" and SMG was suggested by our comparison of EEB-related activations with those elicited by meta-analyses of theory of mind, agency, and imitation inhibition tasks. For a more conclusive assessment of segregation, however, within-subject comparisons of activations associated with the tasks used in the meta-analyses with those related to overcoming EEB are needed. The direct comparison (by means of a conjunction) of EEB with attention reorienting pointed in the same direction, suggesting that overcoming EEB versus overcoming attentional focus is attained by activations that might be centered on different subdivisions of $\mathrm{rTPJ} / \mathrm{rSMG}$. However, in the absence of a statistically significant dissociation from the direct comparison of activation strengths in the two tasks, we cannot rule out the possibility that these neighboring neural populations in rTPJ/ rSMG may be engaged in related computational functions that are only differentially (but not mutually exclusively) recruited in the two contexts. More fine-grained or different types of analyses (focusing on, e.g., resting-state connectivity or multivariate pattern analysis) are needed to clarify this issue.

Notwithstanding these caveats, the possible anatomical segregation tentatively suggested by our findings is further supported by recent results which revealed three subdivisions within rTPJ using structural and functional connectivity measures. One of these subdivisions encompassed the SMG and showed strongest connectivity with areas such as medial cingulate cortex and insular cortex (Mars et al., 2012), areas generally associated with affect regulation (Vogt, 2005; Craig, 2009) and affect sharing (Lamm et al., 2011). In contrast, a more posterior subdivision centered on angular gyrus showed strongest connectivity with areas such as precuneus and medial prefrontal cortex, which have been associated with theory of mind and the suppression of imitative tendencies (Brass et al., 2009; Mar, 2011). This parcellation of rTPJ recently received independent confirmation (Bzdok et al., 2013).

The importance of rSMG in regulating egocentricity was furthermore supported by the TMS findings. Although the limited spatial resolution of TMS might entail that the more posterior division of rTPJ may have also been somewhat affected by the TMS protocol we used, the peak coordinates of the stimulated rSMG spot and the peak coordinates of the other functions in fact showed a considerable spatial distance (from 15 to $26 \mathrm{~mm}$; Table 4). Future investigations should nevertheless directly compare rTMS of rSMG versus more posterior subdivisions of rTPJ to test their distinctive contributions to EEB and other socio-cognitive functions.
The effective connectivity analyses of the fMRI data suggest a possible mechanism by which multisensory integration of self- and other-related information in rSMG may contribute to overcoming egocentricity biases. During incongruent judgments about the other's state, connectivity was increased between rSMG and areas that process sensory information accessible from a proprioceptive, firstperson perspective (i.e., somatosensory cortex, notably only contralateral to the stimulated hand), as opposed to exteroceptive information about another person's sensations and inner states (i.e., visual cortex). The increased interactions of rSMG and both visual and somatosensory brain areas under conditions where EEB occurs might therefore reflect processes that help to detect and disambiguate these types of information, to resolve incongruent information related to the self versus another. This interpretation is in line with previous research on the role of rSMG and adjacent areas in multisensory integration and body ownership (i.e., distinguishing one's physical body from its mental representation). Damage of inferior parietal cortex, including SMG, can produce a variety of disorders associated with distorted body knowledge and self-awareness (Berlucchi and Aglioti, 1997). Recently, it has been demonstrated that experimentally induced out-of-body illusions rely on multisensory integration associated with left and right SMG function (Ionta et al., 2011), in a location almost identical to the one identified in our study. Such illusions demonstrate that the mechanisms of detecting mismatching external and internal signals might not always function properly. Accordingly, our findings speak to a special case of such malfunctions in the socio-affective domain that may lead to egocentrically biased emotion judgments of others.

Interestingly, rSMG so far has not been specifically associated neither with affective (Lamm et al., 2011) nor with cognitive aspects of empathy (Shamay-Tsoory, 2011). This, however, might be explained by the fact that in previous studies, participants usually were in a neutral emotional state without direct sensory or affective stimulation while empathizing with others. Hence, rSMG might only be relevant if self-related affective representations need to be disentangled from other-related ones, such as when being in an incongruent emotional state oneself.

In terms of limitations, we experimentally elicited emotions by a combination of tactile and visual stimulation. Future research with paradigms that elicit emotions through a different set of stimulation will help assure the generalizability of our findings. In addition, to increase statistical homogeneity, we only investigated female undergraduate participants, limiting the validity of the present results to the female population.

\section{Summary and conclusion}

Understanding how egocentrism arises is of increasing importance, as egocentrism and narcissism have been steadily increasing over the last decades, with some scholars even talking about a "narcissism epidemic" (Twenge et al., 2008; Twenge and Campbell, 2009). Egocentricity is also a prominent feature in a variety of mental disorders. Thus, the paradigm we developed may prove immensely useful for diagnosis and research in clinical and developmental settings.

In addition to establishing the existence of EEB in healthy human adults, our study provides insights into the neural mechanisms allowing preventing emotionally biased empathic judgments. The regulation of incongruencies between self- and other-related affective information seems to be supported by a mechanism disentangling self-related proprioceptive and bodily states (which notably are a crucial component of all emotions) from exteroceptive, publicly available information about the other. The observation that EEB increases when either disrupting rSMG or using a shorter stimulation time suggests that these early perceptual integration processes 
and associated regulatory mechanisms might require some time to become effective.

The present results suggest that previous models from social neuroscience based on findings showing that we use first-hand affective neuronal representations as a basis for empathic judgments about affective states of others have to be extended (Bastiaansen et al., 2009; Mitchell, 2009; Singer and Lamm, 2009). Thus, although such simple projection mechanisms based on shared neuronal networks may be used and efficient if oneself is in a neutral or congruent affective state to another, an additional mechanism has to be recruited in cases of incongruency between ones' own and the others' state. Our results suggest that rSMG is subserving such additional mechanisms for self-other distinction in the context of affective social judgments.

\section{References}

Bastiaansen JA, Thioux M, Keysers C (2009) Evidence for mirror systems in emotions. Philos Trans R Soc Lond B Biol Sci 364:2391-2404. CrossRef Medline

Berlucchi G, Aglioti S (1997) The body in the brain: neural bases of corporeal awareness. Trends Neurosci 20:560-564. CrossRef Medline

Brass M, Derrfuss J, von Cramon DY (2005) The inhibition of imitative and overlearned responses: a functional double dissociation. Neuropsychologia 43:89-98. CrossRef Medline

Brass M, Ruby P, Spengler S (2009) Inhibition of imitative behaviour and social cognition. Philos Trans R Soc Lond B Biol Sci 364:2359-2367. CrossRef Medline

Bzdok D, Langner R, Schilbach L, Jakobs O, Roski C, Caspers S, Laird AR, Fox PT, Zilles K, Eickhoff SB (2013) Characterization of the temporo-parietal junction by combining data-driven parcellation, complementary connectivity analyses, and functional decoding. Neuroimage 81:381-392. CrossRef Medline

Corbetta M, Patel G, Shulman GL (2008) The reorienting system of the human brain: from environment to theory of mind. Neuron 58:306-324. CrossRef Medline

Craig AD (2009) How do you feel-now? The anterior insula and human awareness. Nat Rev Neurosci 10:59-70. CrossRef Medline

Decety J, Lamm C (2007) The role of the right temporoparietal junction in social interaction: how low-level computational processes contribute to meta-cognition. Neuroscientist 13:580-593. CrossRef Medline

Duvernoy HM (1991) The human brain: surface, three-dimensional sectional anatomy and MRI. New York: Springer.

Eickhoff SB, Stephan KE, Mohlberg H, Grefkes C, Fink GR, Amunts K, Zilles K (2005) A new SPM toolbox for combining probabilistic cytoarchitectonic maps and functional imaging data. Neuroimage 25:1325-1335. CrossRef Medline

Eickhoff SB, Grefkes C, Fink GR, Zilles K (2008) Functional lateralization of face, hand, and trunk representation in anatomically defined human somatosensory areas. Cereb Cortex 18:2820-2830. CrossRef Medline

Eisenegger C, Treyer V, Fehr E, Knoch D (2008) Time-course of “off-line” prefrontal rTMS effects: a PET study. Neuroimage 42:379-384. CrossRef Medline

Friston KJ (2002) Bayesian estimation of dynamical systems: an application to fMRI. Neuroimage 16:513-530. CrossRef Medline

Friston KJ, Buechel C, Fink GR, Morris J, Rolls E, Dolan RJ (1997) Psychophysiological and modulatory interactions in neuroimaging. Neuroimage 6:218-229. CrossRef Medline

Gitelman DR, Penny WD, Ashburner J, Friston KJ (2003) Modeling regional and psychophysiologic interactions in fMRI: the importance of hemodynamic deconvolution. Neuroimage 19:200-207. CrossRef Medline

Ionta S, Heydrich L, Lenggenhager B, Mouthon M, Fornari E, Chapuis D, Gassert R, Blanke O (2011) Multisensory mechanisms in temporoparietal cortex support self-location and first-person perspective. Neuron 70:363-374. CrossRef Medline

Janowski V, Camerer C, Rangel A (2013) Empathic choice involves vmPFC value signals that are modulated by social processing implemented in IPL. Soc Cogn Affect Neurosci 8:201-208. CrossRef Medline

Kirk RE (1996) Practical significance: a concept whose time has come. Educ Psychol Measure 56:746-759. CrossRef

Kriegeskorte N, Lindquist MA, Nichols TE, Poldrack RA, Vul E (2010) Everything you never wanted to know about circular analysis, but were afraid to ask. J Cereb Blood Flow Metab 30:1551-1557. CrossRef Medline
Lamm C, Decety J, Singer T (2011) Meta-analytic evidence for common and distinct neural networks associated with directly experienced pain and empathy for pain. Neuroimage 54:2492-2502. CrossRef Medline

Mar RA (2011) The neural bases of social cognition and story comprehension. Annu Rev Psychol 62:103-134. CrossRef Medline

Mars RB, Sallet J, Schüffelgen U, Jbabdi S, Toni I, Rushworth MF (2012) Connectivity-based subdivisions of the human right "temporoparietal junction area": evidence for different areas participating in different cortical networks. Cereb Cortex 22:1894-1903. CrossRef Medline

Mitchell JP (2008) Activity in right temporo-parietal junction is not selective for theory-of-mind. Cereb Cortex 18:262-271. CrossRef Medline

Mitchell JP (2009) Inferences about mental states. Philos Trans R Soc Lond B Biol Sci 364:1309-1316. CrossRef Medline

Mitchell JP, Macrae CN, Banaji MR (2006) Dissociable medial prefrontal contributions to judgments of similar and dissimilar others. Neuron 50: 655-663. CrossRef Medline

Morishima Y, Schunk D, Bruhin A, Ruff CC, Fehr E (2012) Linking brain structure and activation in temporoparietal junction to explain the neurobiology of human altruism. Neuron 75:73-79. CrossRef Medline

Nicolle A, Klein-Flügge MC, Hunt LT, Vlaev I, Dolan RJ, Behrens TE (2012) An agent independent axis for executed and modeled choice in medial prefrontal cortex. Neuron 75:1114-1121. CrossRef Medline

O'Brien E, Ellsworth PC (2012) More than skin deep: visceral states are not projected onto dissimilar others. Psych Sci 23:391-396. CrossRef Medline

Poldrack RA (2007) Region of interest analysis for fMRI. Soc Cogn Affect Neurosci 2:67-70. CrossRef Medline

Posner MI, Rueda MR, Kanske P (2007) Probing the mechanisms of attention. In: Handbook of psychophysiology (Cacioppo JT, Tassinary LG, Berntson GG, eds), pp 410-432. Cambridge: Cambridge UP.

Pronin E (2008) How we see ourselves and how we see others. Science 320: 1177-1180. CrossRef Medline

Repacholi BM, Gopnik A (1997) Early reasoning about desires: evidence from 14- and 18-month-olds. Dev Psychol 33:12-21. CrossRef Medline

Rosenthal R, Rubin DB (1982) A simple, general purpose display of magnitude of experimental effect. J Educ Psych 74:166-169. CrossRef

Rossi S, Hallett M, Rossini PM, Pascual-Leone A (2009) Safety, ethical considerations, and application guidelines for the use of transcranial magnetic stimulation in clinical practice and research. Clin Neurophysiol 120:2008-2039. CrossRef Medline

Royzman EB, Cassidy KW, Baron J (2003) "I know, you know": epistemic egocentrism in children and adults. Rev Gen Psych 7:38-65. CrossRef

Santiesteban I, Banissy MJ, Catmur C, Bird G (2012) Enhancing social ability by stimulating right temporoparietal junction. Curr Biol 22:2274-2277. CrossRef Medline

Scholz J, Triantafyllou C, Whitfield-Gabrieli S, Brown EN, Saxe R (2009) Distinct regions of right temporo-parietal junction are selective for theory of mind and exogenous attention. PLoS ONE 4:e4869. CrossRef Medline

Shamay-Tsoory SG (2011) The neural bases for empathy. Neuroscientist 17:18-24. CrossRef Medline

Singer T, Lamm C (2009) The social neuroscience of empathy. Ann N Y Acad Sci 1156:81-96. CrossRef Medline

Spengler S, von Cramon DY, Brass M (2009) Control of shared representations relies on key processes involved in mental state attribution. Hum Brain Mapp 30:3704-3718. CrossRef Medline

Sperduti M, Delaveau P, Fossati P, Nadel J (2011) Different brain structures related to self- and external-agency attribution: a brief review and metaanalysis. Brain Struct Funct 216:151-157. CrossRef Medline

Twenge JM, Campbell WK (2009) The narcissism epidemic: living in the age of entitlement. New York: Free.

Twenge JM, Konrath S, Foster JD, Campbell WK, Bushman BJ (2008) Egos inflating over time: a cross-temporal meta-analysis of the Narcissistic Personality Inventory. J Pers 76:875-902; discussion 903-928. CrossRef Medline

Van Boven L, Loewenstein G (2003) Social projection of transient drive states. Pers Soc Psych Bull 29:1159-1168. CrossRef Medline

Van Overwalle F (2009) Social cognition and the brain: a meta-analysis. Hum Brain Mapp 30:829-858. CrossRef Medline

Vogt BA (2005) Pain and emotion interactions in subregions of the cingulate gyrus. Nat Rev Neurosci 6:533-544. CrossRef Medline

Young L, Dodell-Feder D, Saxe R (2010) What gets the attention of the temporo-parietal junction? An fMRI investigation of attention and theory of mind. Neuropsychologia 48:2658-2664. CrossRef Medline 